UDC 683.519

DOI: $10.20535 /$ SRIT.2308-8893.2018.1.05

\title{
MEDICAL IMAGE SEGMENTATION METHODS OVERVIEW
}

\author{
B.V. CHAPALIUK, Yu.P. ZAYCHENKO
}

\begin{abstract}
This article provides an overview of the modern medical image segmentation methods. The most popular methods such as multi-atlas based methods and deep learning approach are considered in more details. In addition, this article overviews different steps of the multi-atlas based methods (MAS) in detail and shows which modern algorithms and approaches used in different steps of MAS to achieve state of the art results in the medical image segmentation task and how it affects the accuracy of the algorithm. Also, there is a brief description of the modern deep learning algorithms which are used for the medical image segmentation. Such type of algorithm is used as an independent algorithm or as a part of the MAS. Finally, this article summarizes described algorithms and evaluates which approaches promise to improve state of the art result of the medical image segmentation in the future.
\end{abstract}

Keywords: medical image segmentation, multi-atlas based method, deep learning approach

\section{INTRODUCTION}

In the last few years, there is huge progress in the computer vision task with the help of the convolution neural networks $(\mathrm{CNN})$ and an increasing size of the labelled datasets for training. This success is attributed to the ability of the CNN to learn a hierarchical representation of raw input data, without the usage of the handcrafted features. Since the deep learning approaches become widely used in computer vision field, there are lots of the works, which try to apply the same algorithms and approaches for the medical image processing problem. Modern image analysis technologies make good progress in the field of computer vision due to deep learning algorithms and increasing number of available datasets as well.

One of the fundamental problems in the medical image processing is segmentation. It refers to the process of tagging pixel region of interest (ROIs) with biologically meaningful labels, for example, anatomical structures or tissue type. As usual, to make modern deep learning algorithms work there should be a huge amount of data which are manually labelled by the trained expert. Labelling and segmenting dataset are expensive and complicated part of the application of medical image analytics since medical image should be manually labelled by the specialist in the medical area. Also, the labelled result may differ depending on the operator, prone to error, not scalable and hard to reproduce. Furthermore, the quality of dataset depends on the expert performance. Automatic [1] or semiautomatic [2] segmentation algorithms can address these problems by speeding up the process, reducing the cost and time, which should be spent by an expert, offering reliability, repeatability, and scalability.

There are several methods typically used for the medical image segmentation: graph-based optimal image segmentation [3], multi-atlas based methods [4], geometric deformable model approaches [5] and deep learning 
approaches [6]. This article considers the most popular modern approaches for automatic image segmentation such as multi-atlas based methods and deep learning approach. Next chapter will review all the stages of the multi-atlas based methods framework and will consider which approaches and algorithms are used on each stage. The last chapter will briefly overview deep learning techniques, which might be as independent segmentation algorithms or might be a part of the multi-atlas based methods.

\section{MULTI-ATLAS BASED METHODS}

Multi-atlas based segmentation methods (MAS) are the class of methods which aim to automatic segmentation of anatomical structure on the target image by propagating a set of annotations from the set of atlas images to the new coordinates on the novel image through image registration process. Typically, atlas images are manually segmented and annotated by the costly effort of the domain expert who relies on the interactive visualisation software [7,8].

There are several types of the atlas-based methods - probabilistic or parametric methods which build a probabilistic representation of the set of the atlases $[9,10]$ and nonparametric methods which use subset of atlases directly (this type of the atlas-based methods are called multi-atlas based methods) [11, 12].

There is a comparison between parametric and non-parametric methods [13] which gives the evidence that the second type of methods shows better accuracy than the first one. The success of these methods can be found in their possibility to use the best-suited atlas images subset for segmenting each particular target subject. Another explanation can be based on the fact that the registration of each of the several best-suited atlases subsets to the target image is more robust than the single image registration between the probabilistic atlas and the target image. It is evident that the drawback of the atlas subset usage is an increasing requirement to the computation performance. However, it gives more accurate and robust results. Therefore, the class of atlas-based methods should be chosen depending on the application requirements. As far as the multi-atlas based methods show better performance then probabilistic atlas-based method, this article will consider them in more detail.

Formally, the goal of MAS is segmentation of some target image T using a set of atlases $A_{1}, \ldots, A_{m}$, and their corresponded maps label $L_{1}, \ldots, L_{m}$ which values can be 1 or 0 depend on whether voxel $\mathrm{x}$ belongs to the structure of interest (1) or not (0). To achieve it, as usual MAS is involved into the next three steps: atlas selection, image registration, label fusion step.

Additionally, modern algorithms can add some additional steps to the MAS pipeline like online learning [14-17] and post-processing steps [18-21].

\section{Atlas selection step}

In the scope of this step, MAS algorithms select the subset of the atlas images that are the most anatomically similar to the target subject $S_{T}$ in $(1, \ldots, m)$. All available atlas images are not used in MAS for the several reasons: first, this approach will improve computation efficiency of the algorithm which might be very important for the application which has strict time constraints. Second, excluding the 
irrelevant images for the target subject $S_{T}$ would improve final algorithm accuracy.

At the early time in the scope of the atlas selection step the subset of the atlas images are picked up randomly [11]. Under random selection of the atlas images, the accuracy of the algorithm improves with increasing number of the atlases still the number of atlases should not be too high to avoid accuracy worsening by introduced not well-suited candidates. It occurs due to the combining result of the multiple atlases leads to correct the errors of the one individual atlas. This idea is fundamental for the multi-atlas based algorithms.

The MAS algorithm segmentation performance can be improved in comparison with the randomly chosen atlases if the algorithm chooses the set of atlases which best-representing anatomy of the target image. That is because the non-related anatomical characteristic will be filtered out. The main issue, which the atlas selection step is solving, consists of defining a function that reflects the similarity between an atlas $A_{i}$ and the target image $T$. To resolve this task there are lots of the metrics used. The metrics might inlclude the similarity measures on the image intensities or normalized mutual information [22], registration consistancy [23], cross-correlation of intensity values [24].

More recently, there are several works which defined another approach to increase performance of atlas selection step by using clustering [25]. In this paper, the authors propose to define the vector of a pair atlas agreement factor between two atlases and apply the k-means algorithm to this feature vector. Furthermore, the ranking parameter, which evaluates the mean SSD between cluster means, is aplied to the result clusters. Such approach improves the total performance of their MAS algorithm.

On the other hand, atlas selection might be treated as a learning algorithm. Such approach utilises the necessity of the manual segmentation of the atlas image which reduces the cost and effort of the MAS algorithm development and also tends to be more computation efficiently than discussed approach, especially on the large dataset. For example, this paper [26] introduced Neighborhood Approximation Forest, a supervised learning algorithm which is inspired by the random forest tree algorithm [27]. This algorithm can incorporate distances that are defined on semantic information and relate them to the space of appearancebased feature. Another algorithm, which uses the same metrics for evaluating similarity between target image $T$ and atlas image, is SVM which show state of the art result for this step [28].

To sum up, atlas selection step is an important step of MAS which can affect the final algorithm accuracy. To achieve higher accuracy on the segmentation for atlas selection can be used different metrics. However, the most promised approach is the learning algorithms which use distance metrics like SSD. On the other hand, an approach of atlas selection algorithm might vary depending on the image registration algorithm is used and specific application requirement like computation time of segmentation.

\section{Image registration step}

By the registration step in the scope of the MAS is meant the task of establishing a spatial correspondence between the target image and set of atlases. As a result, the labels can be directly propagated. It means that the possible values of labels on each target pixel correspond to the pixel on the target image. 
More formally [4], set of atlases $\bar{A}$ correspondences with the target image $T$ can be described as follows:

$$
L_{i}=\max _{\varphi}\left\{\operatorname{sim}\left(T, \varphi\left(A_{i}\right)-\lambda \operatorname{reg}(\varphi)\right)\right\},
$$

where operation $\mathrm{stm}$ is some similarity measurement term between two images, operation reg is a regularization term, which can control the flexibility of the transformation with constant $\lambda, A_{i}$ - atlas image, $\varphi$ is a spatial transformation.

The result of the label map calculation will be used on the label fusion step according to the computed transformation. More formally, it will be used in form $\widetilde{L}_{l} \equiv \varphi\left(L_{i}\right)$. An extensive review of the existing spatial transformation, similarity function and regularization terms can be found in [29]. An extensive comparison review of the image registration methods on the publicly available datasets can be found in [30].

There are two types of the registration types according to its transformation model: linear and deformable registration [29].

Typically, in MAS one single registration is computed between each atlas and the target image. The usage of the atlas sets tends to improve the performance of the registration algorithm and give more robust result as was discussed at the beginning of the paper.

In conclusion, the registration algorithms map the selected atlases to the target image and create a label map with correspondence between pixels on the atlas images and target image T. Recently, the most popular and accurate methods of image registration are methods which are based on the deformable model.

\section{Label fusion step}

Label fusion is an important part of the MAS algorithm pipeline step. During this step, the final segmentation on the target image is produced. It uses the result mapping between each atlas and the target image $T$ which is got from the image registration step. More formally [4], each atlas $\widetilde{A}_{i}$ and the label map $\widetilde{L}_{i}$ are registered to the target image $T$ after image registration step. The segmentation result on each target voxel $x$ of the target image $T$ evaluated by combining labels on the corresponding voxel location $L_{i}(x)$ in the atlas image. The earliest and simplest algorithm of the label fusion are best atlas selection [31] and majority voting rule $[11,32]$. Specifically, it can be described by the next formula:

$$
\hat{F}(x)=\arg \max _{l \in\{0,1\}}\left\{\sum_{i \in S_{T}} \delta\left(L_{i}(x)=l\right)\right\},
$$

where $\delta$ is the function which returns 1 if the argument is true and 0 otherwise, and $S_{T}$ is the subset of the selected atlases indexes.

Even though the majority voting rule is a simple one, it gives better accuracy than any other method which uses a single atlas for image registration [31]. Nevertheless, in the real-world application some correlation in the error pattern might exist, and therefore, more robust label fusion algorithm is needed to compensate it. 
According to the paper [4] there are three categories of the label fusion methods used in the modern MAS applications: weighted voting approaches, probabilistic approaches, machine-learning based approaches.

Weighted voting approaches. These approaches use weights for evaluate importance of each atlas and select the best one. Weights reflect the similarity between the atlas and the target image and might be global or local. Consequently, there are two types of methods: methods which compute weights based on each atlas independently and the methods that compute weights to minimise correlation between error patterns of atlas pairs [33, 34].

In case of the independent weighting strategy, each atlas is evaluated individually with respect to the weights. Weights represent the local importance of atlas and computed based on the similarity function between the local image patches. Typically, as similarity measurement metrics used cross-correlation, mutual information metrics or the sum of squared differences [33], or an empirical measurement might be used [35]. For example, similarity measurement metrics between local image patches of the target image and atlas can be negative SSD exponent [4]:

$$
\omega_{i}(x)=\exp \left(-\gamma \sum_{y \in N_{X}}\left(T(Y)-A_{i}(y)\right)^{2}\right),
$$

where $\gamma$ is a normalization parameter, $N_{x}$ is the spatial neighborhood which defines the image patches centered at $x$.

As a result, the target label will be computed as follows:

$$
\hat{F}(x)=\arg \max _{l}\left\{\sum_{i \in S_{T}} \omega_{i}(x) \delta\left(L_{i}(x)=l\right)\right\},
$$

where $\omega_{i}(x)$ is weight which denotes $i$-th atlas importance in evaluating the target label at the region location $x, \delta$ is the function which returns 1 if the argument is true and 0 otherwise, and $S_{T}$ is the subset of the selected atlases indexes.

There might be two strategies to denote correspondence between the target image and atlas image: one-to-one correspondence and one-to-many correspondences. The one-to-many correspondence is considered to be better than one-toone correspondences as far as it has better robustness because of considering the atlas labels in the spatial neighbourhood [36].

For the one-to-many correspondence strategy [4], the target label calculation will look as follows:

$$
\hat{F}(x)=\arg \max \left\{\sum_{i \in S_{t}} \sum_{y \in N_{x}} \omega_{i}(x, y) \delta\left(L_{i}(y)=l\right)\right\},
$$

where $\omega_{i}(x, y)$ is weight which denotes estimated segmentation performance for the operation of assigning the atlas label at the point $y \in N_{x}^{\prime}$ to the target label at the point $x, N_{x}^{\prime}$ is spatial neighborhood for potential atlas correspondences search, $\delta$ is the function which returns 1 if the argument is true and 0 otherwise, and $S_{T}$ is the subset of the selected atlases indexes. 
The independent weighting strategy works well when the anatomical image characteristics are equally distributed among the atlases, however, in the realworld usually this assumption does not work, and some anatomical characteristics and features may be overrepresented in the set of atlases. To deal with this problem the joint weighting strategy is used. [37] The main idea behind weighting strategy is to minimise the correlation of participating atlases during the weights choosing step instead of computing them independently. This approach enforces that the most represented anatomical characteristic in the dataset not accumulated in most of the calculated weights.

Probabilistic approaches. These approaches use Bayes` probability rules to select the best one label. There are two core algorithms family: STAPLE algorithm $[38,39]$ that directly estimates parameters performance that best suit to the probabilistic estimate of the target labels, and generative probabilistic model [40] which considers weighted voting rule from a Bayesian perspective.

Machine-learning based approaches. These approaches use supervised learning to evaluate the relationship between the appearance feature and the anatomical feature. [41]. To achieve optimal performance the set of atlases with their corresponding label are used to lean the classification rules. In comparison to the traditional MAS label fusion algorithm, which gets the target labels from the image registration step, machine learning approaches can capture more complex relationships between image and labels. The machine learning approaches will be considered in more detail in the next chapter, where the deep learning approaches will be discussed.

\section{DEEP LEARNING APPROACH}

One of the limitations of the previously discussed methods is the inability of adapting themselves to the data at hand. This means that the power of feature representation might vary across the different kind of image data. The handcrafted feature and representations depend on the expert performance, which may vary between different domain experts. Furthermore, manually feature creation cannot tend to the creation of a complex feature pattern. So far as these problems can be resolved by a deep learning approaches, it gains popularity in the research community. Recently, deep learning has become a hot topic in machine learning [42], computer vision and biomedical image processing [43]. Likewise, deep learning approach with its ability to learn hierarchy from raw data tends to improve the overall performance of the task [44] meanwhile reduce the cost of application development since the manual feature design is not required anymore. In addition, there are the deep learning approaches which outperform the classical methods like MAS with a large margin [45].

The work [43] shows an attempt to use the deep learning approach for the multiple organ detections using the $4 \mathrm{D}$ patient data. The authors used an unsupervised technique to create a feature representation for their data, and after that use them in the probabilistic patch-based methods. They use stacked sparse autoencoder (SSAE) to extract the feature from the dataset.

There is also another work, which extends such approach and uses the stack autoencoders as a part of the MAS and deformable model methods [46]. The authors perform the supervised fine-tuning which is adapted by stacking another 
classification output layer on the top of the encoding part of the SSAE. The result of the work shows that the SSAE outperform other classical methods including the MAS and deformable model methods with handcrafted feature representation.

Another approach for applying deep learning algorithms for the medical image segmentation is to use the power of convolutional neural networks (CNN). In [47] it was proposed the U-Net architecture for the CNN which is an extension of the fully convolution neural network [48]. The authors report that they achieve state of the art results on the two challenging light data sets and tasks: segmentation of neuronal structure in EM stacks and cell segmentation in light microscopy challenge from ISBI cell tracking challenge. Consequently, this CNN has a good performance even in the small datasets.

One of the disadvantages of the U-Net CNN is an ability to work only with $2 \mathrm{D}$ data while most of the clinical data consist of the $3 \mathrm{D}$ volumes. To make the CNN to process the 3D image the V-Net CNN architecture was introduced [49]. This architecture achieves the superior result on the PROMISE12 dataset [50].

After all, the deep learning approaches show the state of the art result and promise to achieve state of the art performance on the most biomedical processing task. These approaches might be used as a part of the classical segmentation approaches, like MAS as well as independently.

\section{CONCLUSION}

This article considers auto-segmentation methods which is one of the most widely used methods in the biomedical image processing. For the last 10 years the multiatlas based methods have been rapidly developed and achieved great performance on the different type of medical data. MAS methods consist of the three main steps: label selection, image registration and label fusion steps. The label selection step selects the most anatomically similar atlases to the target image. It has been shown, that the best approach is to select a subset of the anatomically similar atlases rather than use all available atlases. The registration step builds the map of correspondence between each atlas and the target image. This step has a huge impact on the overall MAS algorithm performance, so that the image registration approach should be chosen carefully according to the medical image processing performance. The label fusion step combines the individual decisions with the multiple atlas to decide which label should be applied to the certain point in the image. The most widely used algorithms for the label fusion are vote weighting, probabilistic weighted vote, based on the Bayes` framework. There are algorithms which use machine learning approach also.

On the other hand, recently the deep learning approach has been largely used in the biomedical image processing field and shown much promising result. There are several CNNs architectures which have achieved state of the art results on the different challenge dataset. Recent development shows that different supervised machine learning and unsupervised learning techniques tend to be used in the different steps of the MAS algorithm to improve the overall accuracy.

\section{REFERENCES}

1. $W u Z$. Segmenting hippocampal subfields from 3T MRI with multi-modality images / Z. Wu, Y. Gao, F. Shi et al. // Segmenting hippocampal subfields from 3T MRI with multi-modality images. - Vol. 43. - 2018. - P.10-22. 
2. You $W$. Semi-automatic segmentation of the placenta into fetal and maternal compartments using intravoxel incoherent motion MRI / W. You, N. Andescavage, Z. Zun, C. Limperopoulos // Medical Imaging. — Vol. 10137. — 2017.

3. Oguz I. LOGISMOS: A Family of Graph-Based Optimal Image segmentation methods / I. Oguz, H. Bogunovic, S. Kashyap et al. // Medical Image Recognition, Segmentation and Parsing Machine Learning and Multiple Object Approaches. - Academic Press, 2016. - P.179-206.

4. Sanroma G. Multiple-atlas segmentation in medical imaging / G. Sanroma, G. Wu, M. Kim, M.A. González et al. // Medical Image Recognition, Segmentation and Parsing. - Academic Press, 2016. - P.231-257.

5. Carass A. An Overview of the Multi-Object Geometric Deformable Model Approach in Biomedical Imaging / A. Carass, J.L. Prince // Medical Image Recognition, Segmentation and Parsing Machine Learning and Multiple Object Approaches. - Academic Press, 2016. — P.259-279.

6. Zhou K. Deep Learning for Medical Image Analysis / K. Zhou, H. Greenspan, D. Shen. - Academic Press, 2017.

7. Criminisi A. Geos: Geodesic image segmentation / A. Criminisi, T. Sharp, A. Blake // Computer Vision - ECCV 2008. ECCV 2008. Lecture Notes in Computer Science. - Springer, 2008. - P.99-112.

8. Heiberg $E$. Design and validation of segment-freely available software for cardiovascular image analysis / E. Heiberg, J. Sjogren, M. Ugander et al. // BMC medical imaging. - Vol. 10. - 2010.

9. Pohl K. Bayesian model for joint segmentation and registration / K. Pohl, J. Fisher, L. Grimson et al. // Neuroimage. — Vol. 31. — 2006. — P. 228-239.

10. Yeo $B$. Effects of registration regularization and atlas sharpness on segmentation accuracy / B. Yeo, M. Sabuncu, R. Desikan et al. // Medical image analysis. Vol. 12. - 2008. - P. 603-615.

11. Heckemann $R$. Automatic anatomical brain MRI segmentation combining label propagation and decision fusion / R. Heckemann, J. Hajnal, P. Aljabar et al. // Neuroimage. - N. 33. - 2006. - P. 115-126.

12. Heiberg E. Design and validation of Segment - freely available software for cardiovascular image analysis / E. Heiberg, J. Sjögren, M. Ugander, M. Carlsson et al. // BMC Medical Imaging. - Vol. 10. - 2010. - P. 1.

13. Babalola $K$. An evaluation of four automatic methods of segmenting the subcortical structures in the brain / K. Babalola, B. Patenaude, P. Aljabar et al. // NeuroImage. - Vol. 47, N. 4. - 2009. - P. 1435-1447.

14. Langerak T. Label Fusion in Atlas-Based Segmentation Using a Selective and Iterative Method for Performance Level Estimation (SIMPLE) / T. Langerak, U. van der Heide, A. Kotte et al. // IEEE Transactions on Medical Imaging. Vol. 29. - 2010. - P. 2000-2008.

15. Hao $Y$. Local label learning (LLL) for subcortical structure segmentation: Application to hippocampus segmentation / Y. Hao, T. Wang, X. Zhang et al. // Human Brain Mapping. - Vol. 35. — 2014. — P. 2674-2697.

16. Wolz R. LEAP: Learning embeddings for atlas propagation / R. Wolz, P. Aljabar, J. V. Hajnal et al., the Alzheimer's Disease Neuroimaging Initiative // NeuroImage. - Vol. 49, N. 2. - 2010. - P. 1316-1325.

17. Chakravarty M. Performing label-fusionbased segmentation using multiple automatically generated templates / M. Chakravarty, P. Steadman, M. Eede et al. // Human brain mapping. - Vol. 34. - 2013. - P. 2635-2654.

18. Yushkevich $P$. Nearly automatic segmentation of hippocampal subfields in in vivo focal T2-weighted MRI / P. Yushkevich, H. Wang, J. Pluta et al. // Neuroimage. - Vol. 53. - 2010. - P. 1208-1224. 
19. Nouranian S. A multi-atlas-based segmentation framework for / S. Nouranian, S. Mahdavi, I. Spadinger et al. // IEEE Transactions on Medical Imaging. Vol. 34. - 2015. - P. 950-961.

20. Wang L. Segmentation of neonatal brain MR images using patch-driven level sets / L. Wang, F. Shi, G. Li et al. // Neuroimage. - Vol. 84. — 2014. — P. 141-158.

21. Asman A. Out-of-atlas likelihood estimation using multi-atlas segmentation / A. Asman, L. Chambless, R. Thompson, B. Landman // Medical physics. Vol. 40, N. 4. - 2013.

22. Studholme C. An overlap invariant entropy measure of 3D medical image alignment / C. Studholme, D.L. Hill, D. Hawkes // Pattern Recognition. — Vol. 32. 1999. - P. 71-86.

23. Heckemann $R$. The mirror method of assessing segmentation quality in atlas label/ R. Heckemann, A. Hammers, P. Aljabar et al. // Biomedical Imaging: From Nano to Macro, 2009. ISBI '09. IEEE International Symposium on. - 2009. P. 1194-1197.

24. Avants B. The optimal template effect in hippocampus studies of diseased populations / B. Avants, P. Yushkevich, J. Pluta et al. // NeuroImage. - Vol. 49, N. 3. - 2010. - P. 2457-2466.

25. Nouranian $S$. A Multi-Atlas-Based Segmentation Framework for Prostate Brachytherapy / S. Nouranian, S.S. Mahdavi, I. Spadinger et al. // IEEE Transactions on Medical Imaging. - Vol. 34, N. 4. - 2015. - P. 950-961.

26. Konukoglu E. Neighbourhood approximation using randomized forests / E. Konukoglu, B. Glocker, D. Zikic, A. Criminisi // Medical Image Analysis. Vol. 17, N. 7. - 2013. - P. 790-804.

27. Liaw A. Classification and Regression by randomForest / A. Liaw, M. Wiener // R news. - Vol. 2, N. 3. - 2002. - P. 18-22.

28. Sanroma G. Learning to rank atlases for multiple-atlas segmentation / G. Sanroma, G. Wu, Y. Gao, D. Shen // IEEE Trans Med Imaging. — Vol. 33. — 2014. P. 1939-1953.

29. Sotiras A. Deformable Medical Image Registration: A Survey / A. Sotiras, D. Christos, P. Nikos // IEEE Transactions on Medical Imaging. - Vol. 32. 2013. - P. 1153-1190.

30. Ou Y. Comparative Evaluation of Registration Algorithms in Different Brain Databases With Varying Difficulty: Results and Insights / Y. Ou, H. Akbari, M. Bilello et al. // IEEE Transactions on Medical Imaging. — Vol. 33. - 2014. - P. 2039-2065.

31. Rohlfing T. Evaluation of atlas selection strategies for atlas-based image segmentation with application to confocal microscopy images of bee brains / T. Rohlfing, R. Brandt, R. Menzel, Jr. CR Maurer // Neuroimage. - Vol. 21. 2004. - P. 1428-1442.

32. Klein A. Automated brain labeling with multiple atlases / A. Klein, B. Mensh, S. Ghosh et al. // BMC Medical Imaging. - Vol. 5. - 2005. - P. 7.

33. Artaechevarria $X$. Efficient classifier generation and weighted voting for atlas-based segmentation: Two small steps faster and closer to the combination oracle / X. Artaechevarria, A. Muñoz-Barrutia, C. Ortiz-de Solorzano // Medical Imaging 2008: Image Processing. - 2008.

34. Wan J. Automated reliable labeling of the cortical surface / J. Wan, A. Carass, S. Resnick, J. Prince // Biomedical Imaging: From Nano to Macro. — 2008. P. 440-443.

35. Zhang D. Confidence-guided sequential label fusion for multi-atlas based / D. Zhang, G. Wu, H. Jia, D. Shen // MICCAI. - Vol. 6893. - 2011. — P. 643-650.

36. Rousseau F. A supervised patch-based approach for human brain labeling / F. Rousseau, P.A. Habas, C. Studholme // IEEE Transactions on Medical Imaging. — Vol. 30, N. 10. — 2011. - P. 1852-1862. 
37. Wang $H$. Regression-based label fusion for multi-atlas segmentation / H. Wang, J. Suh, S. Das et al. // Computer Vision and Pattern Recognition (CVPR), 2011 IEEE Conference on. - 2011. - P. 1113-1120.

38. Warfield S.K. Simultaneous truth and performance level estimation (STAPLE): an algorithm for the validation of image segmentation / S.K. Warfield, K.H. Zou, W.M. Wells // IEEE Transactions on Medical Imaging. — Vol. 23. — 2004. P. 903-921.

39. Asman A.J. Hierarchical performance estimation in the statistical label fusion framework / A.J. Asman, B.A. Landman // Medical Image Analysis. — Vol. 18, N. 7. - 2014. - P. 1070-1081.

40. Sabuncu M. A Generative Model for Image Segmentation Based on Label Fusion / M. Sabuncu, Y. Thomas, K. Leemput et al. // IEEE Transactions on Medical Imaging. - Vol. 29. - 2010. - P. 1714-1729.

41. Wang $H$. Multi-atlas segmentation with learning-based label fusion / H. Wang, Y. Cao, T. Syeda-Mahmood // Machine Learning in Medical Imaging. 2014. - P. 256-263.

42. Bengio $Y$. Representation Learning: A Review and New Perspectives / Y. Bengio, A. Courville, P. Vincent // IEEE Transactions on Pattern Analysis and Machine Intelligence. - Vol. 35, N. 8. - 2013. - P. 1798-1828.

43. Shin Hoo-Chang Stacked Autoencoders for Unsupervised Feature Learning and Multiple Organ Detection in a Pilot Study Using 4D Patient Data / Hoo-Chang Shin, Matthew R. Orton, David J. Collins, Simon J. Doran, Martin O. Leach // IEEE Transactions on Pattern Analysis and Machine Intelligence. - Vol. 35, N. 8. - 2013. - P. 1930-1943.

44. Krizhevsky A. ImageNet Classification with Deep Convolutional Neural Networks / A. Krizhevsky, I. Sutskever, G. Hinton // Advances in Neural Information Processing Systems 25. — Curran Associates, Inc., 2012. - P.1097-1105.

45. Ciresan D. Deep Neural Networks Segment Neuronal Membranes in Electron Microscopy Images / D. Ciresan, A. Giusti, L. Gambardella, J. Schmidhuber // Advances in Neural Information Processing Systems 25. - Curran Associates, Inc., 2012. - P.2843-2851.

46. Guo Y. Deformable MR Prostate Segmentation via Deep Feature Learning and Sparse Patch Matching / Y. Guo, Y. Gao, D. Shen // Deep Learning for Medical Image Analysis. — Academic Press, 2017. — P.221-247.

47. Ronneberger $O$. U-Net: Convolutional Networks for Biomedical Image Segmentation / O. Ronneberger, P. Fischer, T. Brox // Medical Image Computing and Computer-Assisted Intervention - MICCAI 2015. Lecture Notes in Computer Science. - Springer, 2015. - P.234-241.

48. Jia Y. Caffe: Convolutional architecture for fast feature embedding / Y. Jia, E. Shelhamer, J. Donahue et al. // MM '14 Proceedings of the 22nd ACM international conference on Multimedia. - 2014. - P. 675-678.

49. Milletari F. V-Net: Fully Convolutional Neural Networks for Volumetric Medical Image Segmentation / F. Milletari, N. Navab, Sed-Ahmad Ahmadi // 3D Vision (3DV), 2016 Fourth International Conference on. - 2016. - P. 565-571.

50. Litjens $G$. Evaluation of prostate segmentation algorithms for MRI: the PROMISE12 challenge / G. Litjens, R. Toth, W. van de Ven et al. // Medical image analysis. — Vol. 18. - 2014. - P. 1361-8423. 